\title{
The Sprouty-related protein, Spred, inhibits cell motility, metastasis, and Rho-mediated actin reorganization
}

\author{
Kanta Miyoshi ${ }^{1,2}$, Toru Wakioka ${ }^{1}$, Hitomi Nishinakamura ${ }^{1}$, Masaki Kamio ${ }^{1}$, Lu Yang ${ }^{1}$, \\ Makoto Inoue $^{3}$, Mamoru Hasegawa ${ }^{3}$, Yoshikazu Yonemitsu ${ }^{4}$, Setsuro Komiya ${ }^{2}$ and \\ Akihiko Yoshimura*,1
}

${ }^{1}$ Division of Molecular and Cellular Immunology, Medical Institute of Bioregulation, Kyushu University, 3-1-1 Maidashi, Higashi-ku, Fukuoka 812-8582, Japan; ${ }^{2}$ Department of Orthopedic Surgery, Faculty of Medicine, Kagoshima University, 8-35-1 Sakuragaoka, Kagoshima 890-8520, Japan; ${ }^{3}$ DNAVEC Research Inc., Tsukuba, Ibaraki, Japan; ${ }^{4}$ Department of Pathology Graduate School of Medical Sciences, Division of Pathophysiological and Experimental Pathology, Kyushu University, 3-1-1 Maidashi, Higashi-ku, Fukuoka 812-8582, Japan

Sprouty and the Sprouty-related protein, Spred (Sprouty-related Ena/vasodilator-stimulated phosphoprotein homology-1 (EVH1) domain-containing protein), inhibit Ras-dependent extracellular signal-regulated kinase (ERK) signaling induced by a variety of growth factors. Since Sprouty proteins have been shown to inhibit not only ERK activation but also cell migration, we postulated that Spreds also inhibit cellular migration. Using stably highly metastatic LM8 cells infected with the Spred1-Sendai virus vector, we demonstrated that Spred1 inhibits the metastasis of LM8 cells in nude mice. Spred1 overexpression also inhibited migration of cells in vitro in response to chemokines, CCL19 and CCL21. We also found that Spred1 overexpression dissolved actin-stress fibers. Both EVH1 domain and C-terminal Sprouty-related domain were required for actin reassembly. Spred1 and Spred2 suppressed constitutively activated RhoA (V14RhoA)-induced stress fiber formation and serum response factor activation. Spred1 bound to activated RhoA, but not cdc42 and Rac. Spred1 also inhibited chemokine-induced RhoA activation and active RhoA-induced Rho-kinase activation. These data suggest that Spreds are key regulators of RhoA-mediated cell motility and signal transduction. Furthermore, our study suggests that the induction of Spreds could be a novel strategy for preventing cancer cell metastasis.

Oncogene (2004) 23, 5567-5576. doi:10.1038/sj.onc.1207759 Published online 7 June 2004

Keywords: migration; ERK; Sprouty; metastasis; Rho; signal transduction

\footnotetext{
*Correspondence: A Yoshimura;

E-mail: yakihiko@bioreg.kyushu-u.ac.jp

Received 2 January 2004; revised 17 March 2004; accepted 24 March 2004; published online 7 June 2004
}

\section{Introduction}

Drosophila Sprouty is a membrane-associated protein that functions as a feedback inhibitor of the FGF signaling pathway (Hacohen et al., 1998). Drosophila Sprouty can also antagonize epidermal growth factor signaling pathways (Casci et al., 1999), and overexpression of Sprouty can cause phenotypes resembling those of loss, or reduction, of function of FGF and epidermal growth factor signaling pathways (Kramer et al., 1999; Reich et al., 1999). Mouse homologues of Sproutys (mSprouty $1-4)$ are expressed in embryonic and adult tissues such as the brain, heart, kidney, lung, limbs, and skeletal muscle (De Maximy et al., 1999; Minowada et al., 1999; Tefft et al., 1999; Hashimoto et al., 2002). The physiological functions of mSproutys, however, have not been well established. We and others have shown that mSprouty 2 and mSprouty4 inhibit vascular endothelial growth factor-induced extracellular signalregulated kinase (ERK) activation and vascular branching and sprouting of vessels (Impagnatiello et al., 2001; Lee et al., 2001; Cabrita and Christofori, 2003; Sasaki et al., 2003). Since mSproutys inhibit not only the proliferation but also the migration of endothelial cells (Lee et al., 2001) as well as Hela cells (Yigzaw et al., 2001), mSproutys may modulate cytoskeletal organization.

We recently cloned the Spred1 and Spred2 (Sproutyrelated protein with Ena/vasodilator-stimulated phosphoprotein homology-1 (EVH1) domain) as a physiological endogenous inhibitor of the Ras/Raf-1/ERK pathway by interacting with Ras and Raf-1 (Wakioka et al. 2001; Kato et al., 2003). Spred contains the Cterminal Sprouty-like cysteine-rich domain (SPR-domain) and N-terminal EVH1 domain. The EVH1 domain is an interaction module found in several proteins implicated in actin-based cell motility. EVH1 domains bind the consensus proline-rich motif FPPPP and are required for targeting the actin assembly machinery to sites of cytoskeletal remodeling (Prehoda et al., 1999). Although the Spred EVH1 domain is distantly related to other EVH1 domains (Callebaut 
et al., 1998), the relationship between Spred and cytoskeleton is interesting to investigate.

The actin cytoskeleton plays an important role in defining cell shape and cell morphology and in orchestrating cell migration, axon guidance, phagocytosis, and cytokinesis. In fibroblasts, one of the major actin cytoskeletal structures is stress fibers. The formation of stress fibers is known to be induced by the small GTPase Rho in response to certain extracellular signals in the serum, such as lysophosphatidic acid (Ridley and Hall, 1992a). This appears to be mediated by Rock (Rhokinase/ROK alpha) (Ishizaki et al., 1996; Leung et al., 1996; Amano et al., 1997). Since Spred may relate to the cell motility and cytoskeleton, we analysed the effect of Spred on cell motility and actin reorganization. We demonstrated that Spred1 inhibits the metastasis and migration of LM8 cells. We also found that Spred1 overexpression inhibited actin-stress fiber remodeling. Spred associated with RhoA and suppressed Rhodependent signaling. Our study provides a new insight into the function of Spred/Sprouty family proteins on cell motility.

\section{Results}

\section{Spred and Sprouty suppress tumor metastasis}

To elucidate the effect of Spred on cell motility, we expressed Spred in the highly metastatic mouse osteosarcoma cell line LM8. Spred1 was overexpressed in LM8 cells using a Sendai virus vector $(\mathrm{SeV})$ carrying the Spred 1 gene. The recently developed $\mathrm{SeV}$ possesses very high infection efficiency, like an adenovirus vector, but the expression of the carrying gene persists much longer than with an adenovirus (Yonemitsu et al., 2000). We also constructed the $\mathrm{SeV}$ carrying Sprouty2 for comparison.

First, we compared the effect of Spred1 and Sprouty2 on ERK activation and cell proliferation. As shown in Figure 1a, overexpression of Spred1 and Sprouty2 in LM8 cells suppressed FGF-induced ERK activation, while LacZ infection did not affect the phosphorylation of ERK (Figure 1a, upper panel). As reported previously, Sprouty2 did not inhibit EGF-induced ERK activation, but Spred 1 efficiently suppressed it (Figure 1a lower panel). Both Spred1 and Sprouty2 also suppressed serum-induced ERK activation (data not shown). Then, we compared the cell proliferation. Growth rate of LM8 cells infected with the LacZ virus (LacZ) was almost similar to noninfected parental LM8 (-) cells (Figure 1b). Overexpression of Spred1 and Sprouty2 partially (about $50 \%$ reduction) suppressed proliferation (Figure 1b, Spred1 and Sprouty2). However, this suppressive effect was not as evident as that in previous reports using Hela cells and endothelial cells (Impagnatiello et al., 2001; Lee et al., 2001). This could be due to a low dependence of the growth of LM8 cells on ERK, since the MEK inhibitor PD98059 only partially suppressed the proliferation of LM8 cells (data not shown).

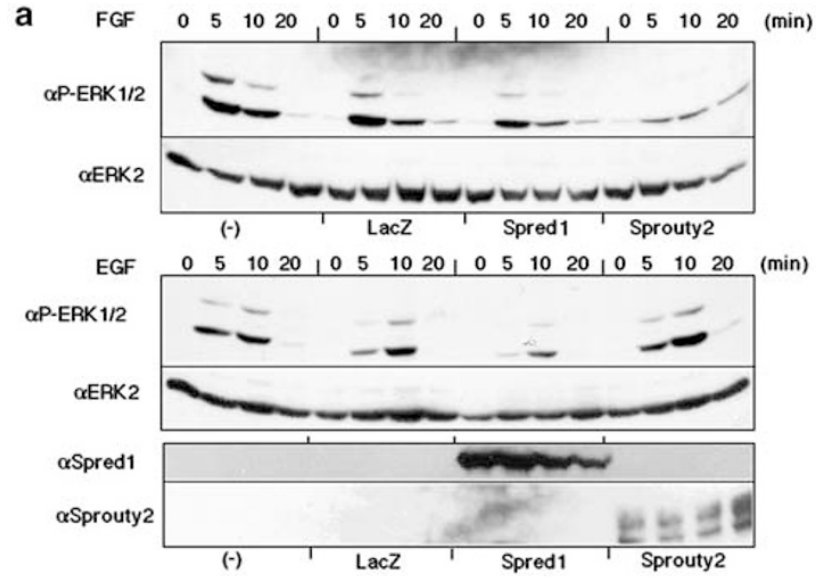

b

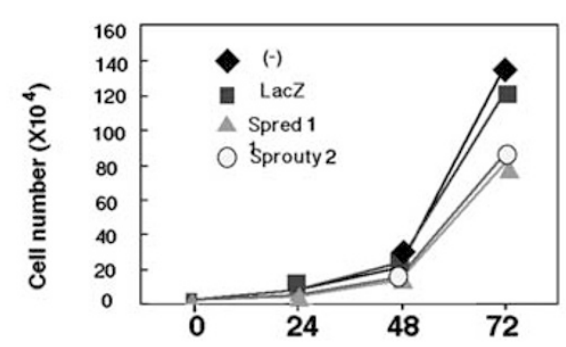

Figure 1 Spred1 and Sprouty2 inhibit LM8 cell proliferation and phosphorylation of ERK1/2. (a) LM8 cells were infected with a Sendai virus (at an MOI =2) carrying Spred1, Sprouty2, and LacZ. More than $95 \%$ of cells were infected judged by $\beta$-galactosidase staining. At 2 days after infection, cells grown in six-well dishes were cultured in $0.5 \%$ CS for $24 \mathrm{~h}$, then stimulated with FGF or EGF $(50 \mathrm{ng} / \mathrm{ml})$ for indicated periods. Total cell extracts were blotted with indicated antibodies. (b) LM8 cells were infected with a Sendai virus carrying Spred1, Sprouty2, or lacZ and then plated into $35 \mathrm{~mm}$ dishes. Cell numbers were counted daily under normal growth conditions. Data represent means of triplicate measurements

Next, we injected LM8 cells after infection of LacZ, Spred1, and Sprouty2 into nude mice subcutaneously. Tumor growth was strongly suppressed by the overexpression of Spred1 and Sprouty2 (data not shown). Although we could not observe lung metastasis in this system, metastasis in the liver efficiently occurred when parental or LacZ-infected LM8 cells was used. Liver metastasis was rarely observed after injection of cells expressing Spred1 or Sprouty2 (Figure 2a). The low metastasis of LM8 cells expressing Spred1 or Sprouty2 could be due to slow proliferation of tumors. Therefore, we directly injected tumor cells intravenously. As shown in Figure $2 b$, strong liver metastasis was observed by the injection of LacZ-infected cells, while liver metastasis was not observed in mice administrated with LM8 cells expressing Spred1 or Sprouty2. These data raise the possibility that both Spred and Sprouty2 were able to suppress the metastasis of tumor cells.

\section{Spred and Sprouty suppress cell migration}

To investigate the role of Spred1 and Sprouty2 on cell migration, we carried out a wound-healing assay 


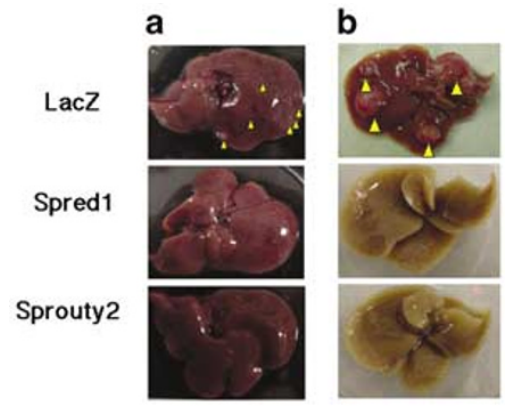

Figure 2 Spred 1 and Sprouty2 inhibit LM8 cell growth and metastasis in the livers of nude mice. (a) LM8 cells $\left(1 \times 10^{7}\right.$ cells $)$ infected with the Sendai virus carrying lacZ, Spred1, and Sprouty2 at an $\mathrm{MOI}=2.0$ were injected subcutaneously into the flanks of nude mice. Tumor growth was monitored for 3 weeks. Arrows indicate visible tumors in the livers. (b) LM8 cells $\left(1 \times 10^{7}\right.$ cells $)$ infected with the Sendai virus were injected intravenously. After 3 weeks, the livers were examined

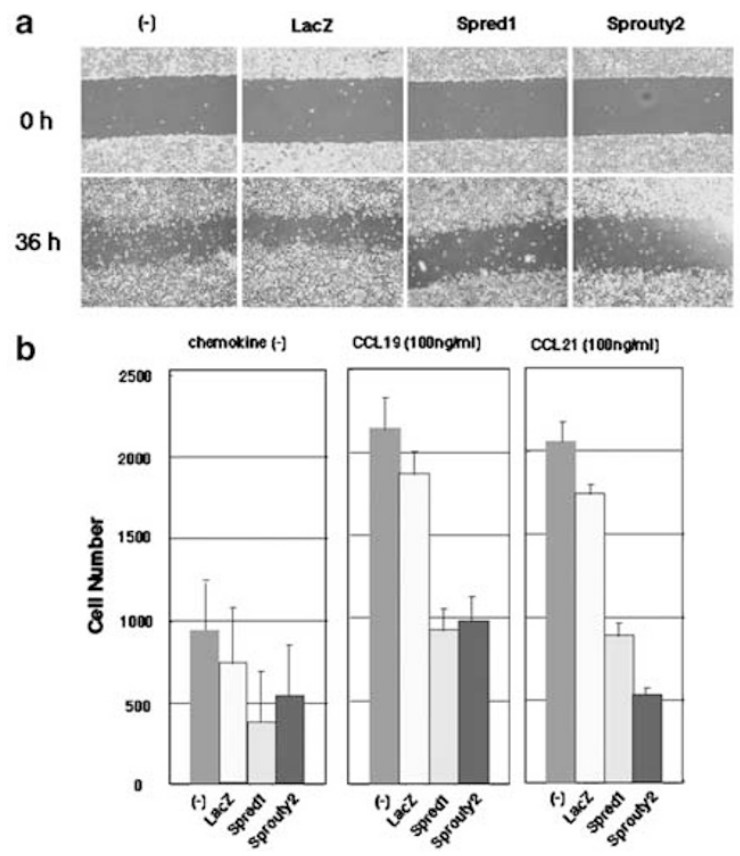

Figure 3 Spred 1 and Sprouty2 suppress LM8 cell migration. (a) Wound-healing assay. LM8 cells expressing LacZ, Spred1, and Sprouty2 grown to subconfluence were scraped with a sharp edge to make a cell-free area $(0 \mathrm{~h})$. Cells were cultured in the presence of mitomycin $\mathrm{C}$ for $36 \mathrm{~h}$ into the scraped area were observed. (b) Chemotaxis assay. Cells infected with the Sendai virus carrying lacZ, Spred1, or Sprouty2 were plated on the upper side of transwells (35000 cells/well). After CCL19 or CCL21 was added to the lower chamber, cells were allowed to migrate overnight at $37^{\circ} \mathrm{C}$. Cells that migrated through the membrane were counted. Data represent means \pm s.d. of triplicate measurements

(Figure 3a) and a chemotaxis assay (Figure 3b). As shown in Figure 3a, LM8 cells infected with LacZ, Spred1, or Sprouty2 grown to subconfluence were scraped with a sharp edge to make a cell-free area. Experiments were carried out in the presence of mitomycin $\mathrm{C}$ to prevent cell proliferation. Cells migrating into the scraped area were observed within $36 \mathrm{~h}$ in noninfected or LacZ-infected cells, whereas Spred1- or Sprouty2-infected cells did not migrate into the scraped area. We also examined cell motility by using a Boyden chamber assay (Figure 3b). Both Spred1 and Sprouty2 inhibited cell migration in response to chemokines, CCL19 and CCL21. It has been reported that ERK pathway is involved in chemokine-mediated cell migration (Bonacchi et al., 2001). Although these two chemokines weakly activated ERK, Spred 1 and Sprouty2 overexpression did not inhibit ERK activation by CCL19 and CCL21 (data not shown). These data suggest that Spred1 and Sprouty2 suppressed cell motility ERK independently.

\section{Effects of Spred1 on stress fiber formation}

Then, we focused on defining the molecular mechanism of the suppression of cell motility by Spred1. We examined the effect of Spred1 expression on the actin cytoskeleton. As shown in Figure 4, both NIH3T3 cells $(\mathrm{a}-\mathrm{h})$ and LM8 cells $(\mathrm{i}-\mathrm{n})$ contain abundant stress fibers and are attached tightly to the substrates. Forced expression of Spred 1 and Spred 2 resulted in a marked loss of actin-stress fiber in NIH3T3 cells (Figure 4, a-c) and LM8 cells (Figure 4m and n). Disruption of stress fiber formation requires both an N-terminal EVH1 domain and a C-terminal SPR domain of Spred1, since $\Delta \mathrm{N}$ and $\Delta \mathrm{C}$ mutant Spred 1 showed little effect on stress fiber formation (Figure 4, e-h).

Since the formation of actin-stress fibers is regulated by the small GTP-binding protein Rho, Spred1 may inhibit Rho signaling. Therefore, we examined the effect of Spred 1 on constitutively activated Rho-induced stress fiber formation by cotransfection of V14RhoA and Spred1. Hela cells were used in this experiment because Rho-induced stress fiber formation was clearly observed. The constitutively active form V14RhoA induced many stress fibers (Figure 5a and b). However, coexpression of Spred1 reduced this stress fiber formation, and actin fibers were localized in the periphery of cells (Figure 5c and d); the inhibition of Rho-induced stress fiber formation depended on the N-terminal EVH1 domain and C-terminal Sprouty domain (Figure 5, e-h).

\section{Spred inhibits Rho-dependent signaling}

To confirm the inhibitory effect of Spred 1 on RhoA, we examined serum response factor (SRF) functions, which are regulated by RhoA. SRF regulates both cellular immediate-early genes and genes expressed in skeletal, smooth, and cardiac muscle RhoA dependently (Hill et al., 1995; Arsenian et al., 1998; Carnac et al., 1998; Wei et al., 1998). SRF activity is also mediated by changes in actin reorganization, whereas the regulation of actin dynamics is necessary for the serum induction of SRF target genes (Sotiropoulos et al., 1999). Both c-fos promoter and SRE reporter are dependent on SRF. As shown in Figure 6, Spred1 suppressed the serum and 

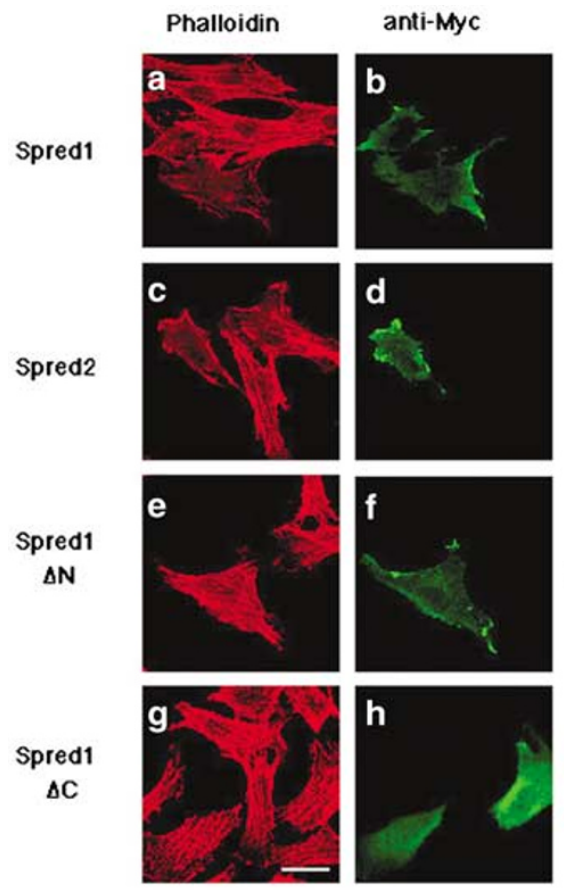

Figure 4 Disruption of stress fibers by Spred1 overexpression. NIH3T3 cells $(\mathbf{a}-\mathbf{h})$ were transfected with pcDNA3 containing wildtype Myc-Spred1 (a, b), wild-type Myc-tagged Spred2 (c, d), Myc-tagged N-terminal EVH1 domain-deleted $(\Delta \mathrm{N})$ Spred1 $(\mathbf{e}, \mathbf{f})$, and Myc-tagged C-terminal SPR domain-deleted $(\Delta \mathrm{C})$ Spred1 (g, h). At $48 \mathrm{~h}$ after transfection, cells were fixed and Myc-tagged Spred was stained with FITC-conjugated secondary antibody $(\mathbf{b}, \mathbf{d}, \mathbf{f}, \mathbf{g})$ in combination with rhodamine-conjugated phalloidin $(\mathbf{a}, \mathbf{c}, \mathbf{e}, \mathbf{h})$. LM8 cells infected with the Sendai virus carrying Spred 1 or lacZ were examined by anti-Myc antibody immunofluorescence and rhodaminephalloidin staining. The results shown are representative of at least three independent experiments. All photographs were taken at the same magnification

V14RhoA-dependent c-fos promoter activation (Figure $6 \mathrm{a}-\mathrm{c}$ ) as well as the SRE reporter activation (Figure 6d). Both $\Delta \mathrm{N}$ and $\Delta \mathrm{C}$ Spred 1 had little effect on c-fos promoter activation, suggesting that EVH1 and SPR domains were necessary for the suppression of SRF activation (Figure 6b).

The interaction between RhoA and Spred was confirmed by using transient overexpression system (Figure 7a). Myc-tagged Spred1 was co-immunoprecipitated with Flag-tagged RhoA, but not Rac or cdc42. The interaction between transfected Spred 1 and active RhoA (V14RhoA) was also confirmed by immunofluorescence microscopy (Figure 7b) and immunoprecipitation (IP) (Figure 8a). Spred1 was localized in the plasma membrane, while V14RhoA was present in both the cytoplasm and the membrane. Functional RhoA is thought to be on the plasma membrane alone (Kranenburg et al., 1997). In the plasma membrane, Spred 1 and RhoA were colocalized (Figure $7 \mathrm{~b}$ ). We also confirmed interaction between V14 RhoA and Spred2 when they were overexpressed in 293 cells (Figure 7c). Interaction between endogenous RhoA and Spred 2 was further examined in Hela cells, since Spred2 was rich in Hela cells and our anti-Spred2 antibody, but not anti-Spred1 antibody worked for IP. As shown in Figure 7d, after IP, endogenous Spred 2 was identified to be an approx. 40-kDa protein and endogenous RhoA was co-immunoprecipitated.
Inhibition of RhoA and downstream activation by Spredl

We then further examined the effect of Spred1 on RhoA activation. First, we examined whether Spred1 interacts with inactive form of RhoA (N19RhoA). N19RhoA could not be detected in Spred1 immunoprecipitates because of dense IgG band; however, V14RhoA binding was detected in the immunoprecipitates with anti-Spred1 (Figure 8a). In contrast, MycSpred-1 could not be precipitated with N19RhoA, while it was precipitated with V14RhoA (Figure 8a). These data indicate that Spred 1 preferentially interacts with activated form of RhoA, probably because only active form of RhoA localizes on the plasma membrane.

Then, we examine the effect of Spred1 on RhoA activation. Using GST-Rho-binding domain pulldown assay, we demonstrated that Spred 1 overexpression partially inhibited CCL19-induced RhoA activation (Figure 8b). Rho-kinase Rock is one of the downstream of activated Rho. We also examined the effect of Spred1 on kinase activation of Rock by V14RhoA. As shown in Figure 8c, Spred1 inhibited V14RhoA-induced Rock activation. These data suggest that Spred1/RhoA interaction inhibits both generation of GTP-RhoA and interaction between activated RhoA and downstream effector molecules such as Rock. 


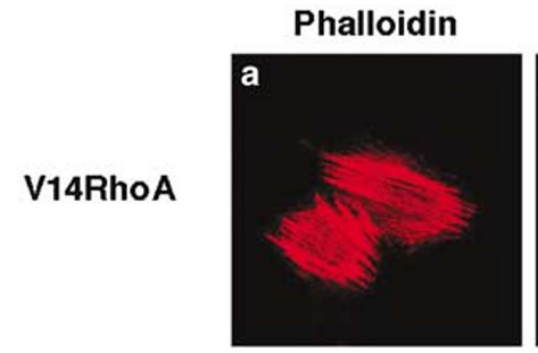

Phalloidin
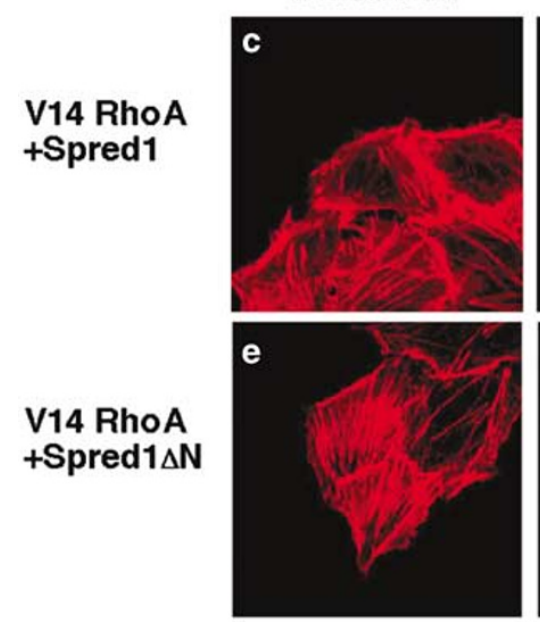

V14 RhoA
+ Spred1 $\triangle N$

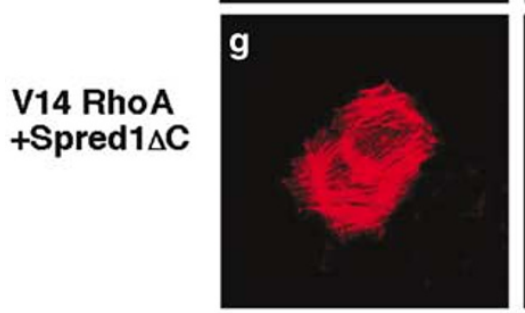

Figure 5 Effects of Spred on V14RhoA-induced stress fiber formation. Hela cells were cotransfected with the Flag-V14RhoA alone $(\mathbf{a}, \mathbf{b})$ or with Myc-Spred 1 wild-type $(\mathbf{c}, \mathbf{d})$, the N-terminal truncated $(\mathbf{e}, \mathbf{f})$, and the C-terminal truncated mutants $(\mathbf{g}, \mathbf{h})(2.0 \mu \mathrm{g}$ total plasmid). At $48 \mathrm{~h}$ after transfection, cells were fixed and then examined by fluorescence microscopy for Flag (b) or Myc $(\mathbf{d}, \mathbf{f}, \mathbf{h})$ immunostaining and rhodamine-phalloidin staining $(\mathbf{a}, \mathbf{c}, \mathbf{e}, \mathbf{g})$

\section{Discussion}

We have isolated Spreds as suppressers of Ras/Raf/ ERK signaling. In this study, we added another function of Spred; overexpression of Spred inhibited tumor metastasis, cell migration, and Rho-dependent stress fiber formation. The Spred-related protein, dSprouty, inhibits the formation of tracheal branches during Drosophila development, and mammalian Sprouty homologues inhibit sprouting and branching of blood vessels during angiogenesis (Lee et al., 2001). Furthermore, mSprouty2 is shown to inhibit not only the proliferation but also the cell migration of Hela cells when overexpressed (Yigzaw et al., 2001). We also confirmed that Sprouty2 inhibited metastasis and cell migration in LM8 cells. However, the molecular mechanism of the suppression of cell migration by Sprouty has not been proposed. In this study, we
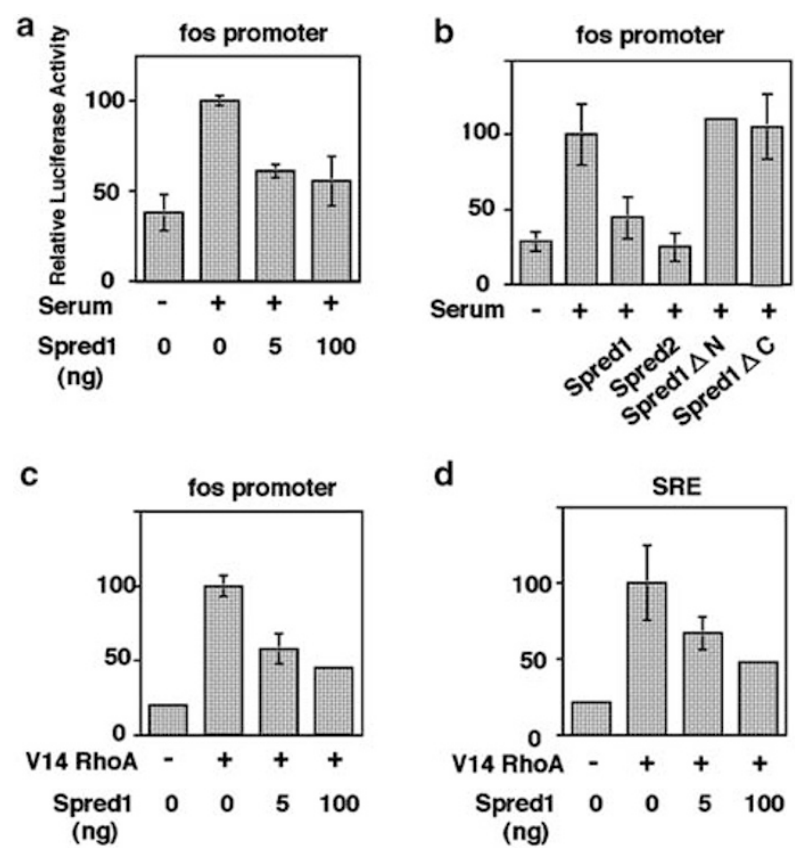

Figure 6 Spred 1 inhibits SRF activity. The 293 cells were transfected with the c-fos promoter luciferase plasmid $(\mathbf{a}, \mathbf{b}, \mathbf{c})$ or SRE reporter plasmid (d) and indicated plasmids. Cells were starved in serum-free DMEM for $20 \mathrm{~h}$ and then unstimulated or stimulated with $10 \%$ calf serum for $6 \mathrm{~h}(\mathbf{a}, \mathbf{b})$. In $(\mathbf{c}, \mathbf{d})$, cells were transfected with reporter plasmids, V14RhoA expression vector and indicated amount of Spred 1 plasmid, then reporter activity was measured. Data represent means \pm s.d. of triplicate measurements

demonstrated that Spred has a similar function to Sproutys. Like Sprouty2, Spred1 inhibited cell migration and tumor metastasis.

Importantly, we showed that Spred1 and Sprouty2 inhibited not only serum- or growth factor-induced but also chemokine-induced cell migration. Chemokines activate the Rho, Rac, and cdc42 pathways through G-protein-coupled receptors. Since Spreds and Sproutys are believed to regulate receptor tyrosine kinases, our study extends the novel function of Spred/Sprouty family proteins. Since Spred suppressed Rho-mediated signaling, we postulate that the effect of Spred on chemokine-dependent cell migration was due to the suppression of Rho.

However, we could not rule out the possibility that Spreds inhibited cell migration by suppressing Ras signaling. Numerous reports indicated that Ras regulates not only cellular proliferation and differentiation but also actin cytoskeletal rearrangement. The microinjection of V12Ras has been reported to induce stress fiber in Swiss 3T3 cells (Ridley et al., 1992b). Rastransformed breast cancer cells display a fibroblastic morphology with decreased cell-cell adhesions but increased focal adhesions and stress fibers; some of these characteristics are thought to be due to activated Rho (Kinch et al., 1995; Zhong et al., 1997). In contrast, Rat1 cells expressing V12Ras- or Ras-transformed fibroblasts showed a marked dissolution of stress fibers and focal adhesions (Khosravi-Far et al., 1994; Izawa 
a

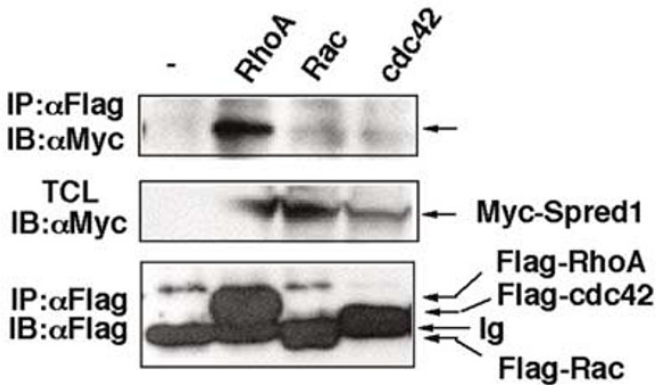

b

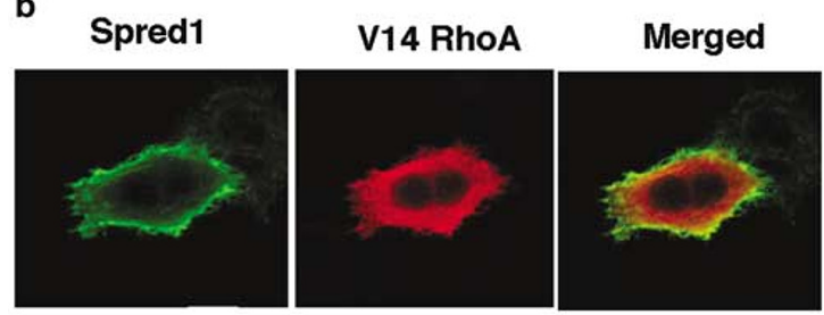

c

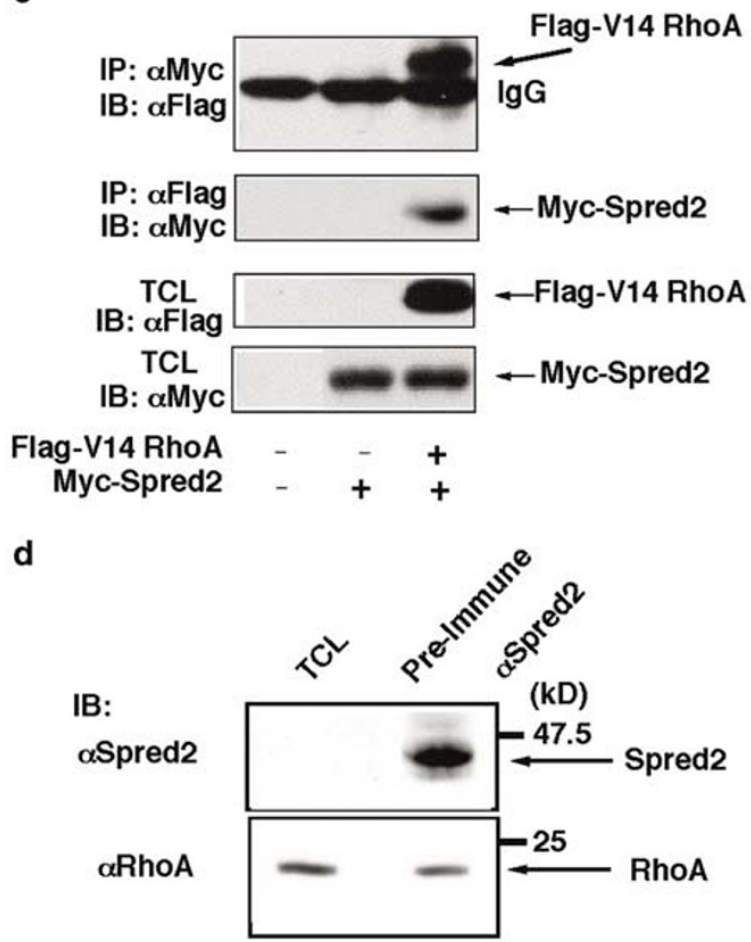

Figure 7 Interaction of Spred and RhoA. (a) Myc-tagged Spred1 and Flag-tagged wild-type RhoA, Rac, or cdc42 were transiently expressed in 293 cells. After IP with anti-Flag antibody, immunoprecipitates or total cell extracts (TCL) were blotted (IB) with indicated antibodies. (b) Immunolocalization of V14RhoA and Spred1. Hela cells were transfected with the Flag-V14RhoA and Myc-Spred1. Cells were fixed and Flag-tagged and Myc-tagged proteins were stained with secondary antibodies conjugated with Alexia (red) and FITC, respectively. (c) Interaction between active RhoA and Spred2. Myc-tagged Spred2 and Flag-tagged V14 RhoA were transiently expressed in 293 cells. Immunoprecipitates with anti-Myc or anti-Flag antibodies or TCL were blotted with indicated antibodies. (d) Interaction of endogenous Spred2 and RhoA. Endogenous Spred2 was immunoprecipitated with preimmune serum or anti-Spred 2 antibody from $1 \times 10^{8}$ Hela cell extracts and then immunoblotted with the indicated antibodies. Blots of TCL are shown on the left et al., 1998). This discrepancy is thought to be due to the regulation of Rho activity by Ras. Ras may regulate the activity of Rho through at least two pathways: the immediate and direct activation of Rho and the relatively late and indirect inactivation of Rho (Izawa et al., 1998). Spred may regulate the actin cytoskeletal rearrangement by inhibiting the Ras/MAP kinase signaling pathway. However, since Spred inhibits Raf kinase activation rather than Ras activation by binding to the Ras/Raf complex (Wakioka et al., 2001), it is unlikely that Spred regulates Rho activity through Ras activity modulation.

We showed that Spred inhibited Rho-mediated signals, stress fiber formation, and SRF activation. However, a downstream target of Rho/Spred is not clear at present. Rock is an attractive target of Rho/Spred complex, since Rock is a target of Rho and responsible for stress fiber formation by Rho (Amano et al., 2000). Our preliminary data (Figure 8) suggest that Spred suppressed stress fiber formation by inactivating the Rho/Rock pathway. Since Spred can directly interact with RhoA, Spred may inhibit Rock activation by interaction with the Rho/Rock complex from the analogy of interaction between Ras/Raf complex. However, further study is necessary to define the clear mechanism of suppression of Rock activation. As seen in our previous report, Spred did not affect EGFdependent membrane ruffling and microspike formation in 293 cells; therefore, Spred might specifically suppress Rho signaling among the Rho family of small GTPases. This is consistent with the failure of binding of Spred 1 to Rac and cdc42 (Figure 7a). Therefore, we propose that the suppression of cell migration and metastasis by Spred is due to the inhibition of Rho. However, since cell migration is a complicated cellular event, the suppression of Ras and Rho could be a part of the mechanisms of the suppression of metastasis and cell migration by Spred.

In contrast, the mechanism of the suppression of cell migration by Sproutys is not clear. To date, Sproutys have been demonstrated to be important in regulating FGF and EGF signaling. Human Sprouty-2 colocalized with microtubules in unstimulated cells and translocated rapidly to membrane ruffles, induced by the small GTPbinding protein, Rac, upon EGF stimulation (Lim et al., 2000). Yigzaw et al. (2001) proposed that Sprouty2 mediates its antimigratory actions by modulating the activity or intracellular localization of protein-tyrosine phosphatases, including PTP1B. Using Hela cells, they showed that overexpression of Sprouty2 resulted in an increase in PTP1B amount and activity in the soluble fraction of cells without an increase in the total amount of cellular PTP1B. However, it is not clear how PTP1B regulates cell migration. Like Spred, Sprouty may not only suppress the Ras/MAP kinase pathway but also regulate actin cytoskeletal rearrangement by regulating Rho.

The antimitogenic effect of Sproutys and Spreds was not very strong in LM8 cells (Figure 1b). However, the overexpression of Spred1 and Sprouty2 suppressed tumor growth and metastasis in vivo (Figure 2). The 
a

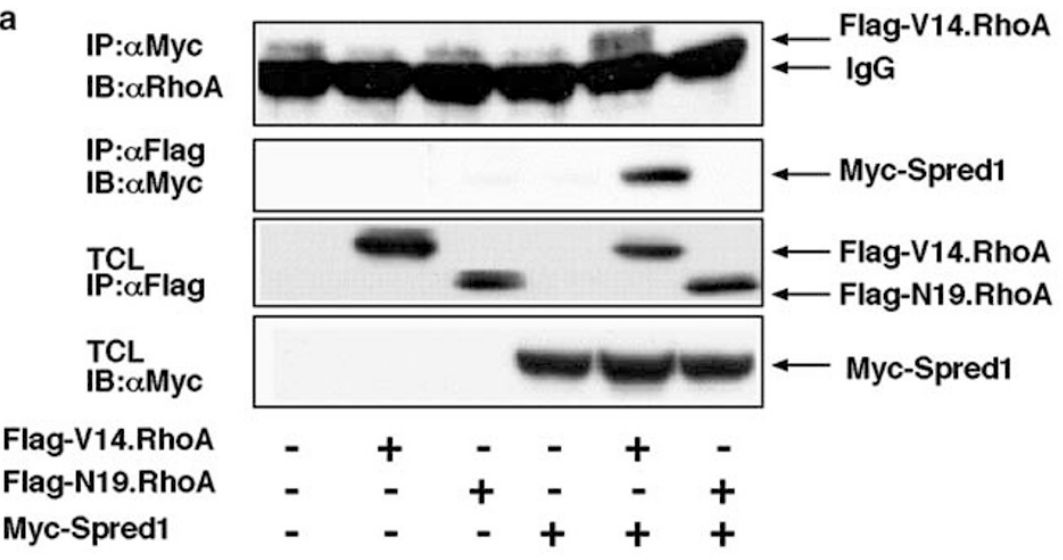

b

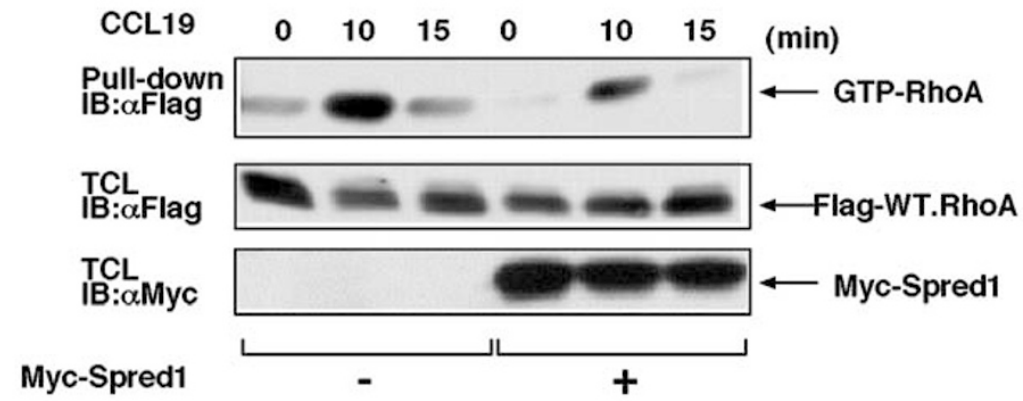

C

Rho kinase assay

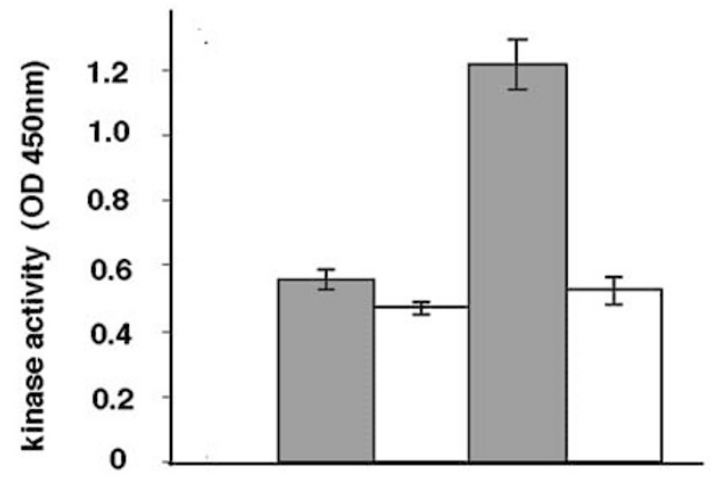

Flag-V14RhoA

Myc-Spred1

Myc-Rock

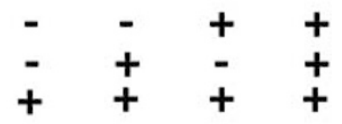

Figure 8 Inhibition of RhoA activity by Spred1. (a) Interaction of activated or inactivated forms of RhoA with Spred1. Myc-tagged Spred 1 and Flag-tagged V14 RhoA or Flag-tagged N19 RhoA were transiently expressed in 293 cells. Immunoprecipitates with antiMyc or anti-Flag antibodies or total cell extracts (TCL) were blotted with indicated antibodies. N19 RhoA could not be detected in the anti-Myc immunoprecipitates because of dense IgG band. (b) Suppression of generation of GTP-bound form of RhoA by Spred1. 293 cells were transfected with Flag-tagged wild type RhoA with or without Spred1. After stimulation of with CCL19 (100 ng/ml) for indicated periods, GTP-form of RhoA was pull down with GST-Rho binding domain of Rhotekin (pull-down) and detected with antiFlag antibody. TCL were also blotted with anti-flag or anti-myc antibodies. (c) Suppression of Rock activity by Spred1. 293 cells were transfected with Myc-tagged Rock together with V12 RhoA and Spred1. After IP with anti-Myc antibody, the immunoprecipitates were subjected to Rho-kinase (Rock) assay. Rho-kinase activity was measured as described in Materials and methods. Data represent means \pm s.d. of triplicate measurements

mechanism of reduced tumor progression by Spred1 and Sprouty 2 overexpression is not clear at present. However, such antitumorigenic and antimetastatic effect of Spred and Sprouty could be a new therapeutic way for cancer treatment. A novel therapeutic target for tumor growth and metastasis will be obtained by defining the molecular mechanism of the action of Spreds and Sproutys. 


\section{Materials and methods}

\section{Cell culture}

Mouse fibroblast NIH3T3 cells, osteosarcoma LM8 cells, and human embryonic kidney 293 cells were cultured in DMEM supplemented with $10 \%$ calf serum (CS). Human cervical carcinoma Hela cells were cultured in DMEM supplemented with $10 \%$ fetal bovine serum (FBS).

\section{Plasmids}

pcDNA3-Myc-tagged Spred1 and Spred2 and deletion mutants were described previously (Wakioka et al., 2001). Human wild-type RhoA, V14RhoA, Rac1, and Cdc42 (gifts from Dr Kaibuchi, Nagoya University) were subcloned into pcDNA3 with a six-repeated Myc-tag or pCMV2 with Flagtag at the N-terminus. The c-fos promoter luc were described previously (Joo et al., 2004). SRE reporter plasmid was purchased from Clontech.

\section{Construction of the recombinant $\mathrm{SeVS}$}

Recombinant SeVs were constructed as described (Kato et al., 1996; Sakai et al., 1999; Yonemitsu et al., 2000). In brief, 18 bp of spacer sequence $5^{\prime}$-(G)-CGGCCGCAGATCTTCACG-3' with a NotI restriction site (underlined) was inserted between the $5^{\prime}$ nontranslated region and the initiation codon of the nucleoprotein $(\mathrm{N})$ gene. This cloned $\mathrm{SeV}$ genome also contains a self-cleaving ribosome site from the antigenomic strand of the hepatitis delta virus. The entire cDNA coding Spred1, mSprouty2, and lacZ was amplified by PCR, using their specific primers with a NotI site and new sets of $\mathrm{SeV}$ E and $\mathrm{S}$ signal sequence tags for an exogenous gene, and then inserted into the Not I site of the cloned genome. The entire length of the template $\mathrm{SeV}$ genome, including exogenous genes, was arranged in multiples of six nucleotides (the so-called 'rule of six') (Kolakofsky et al., 1998). The template $\mathrm{SeV}$ genome with an exogenous gene and plasmids encoding $\mathrm{N}, \mathrm{P}$, and $\mathrm{L}$ genes (plasmid pGEM-N, pGEM-P, and pGEM-L, respectively) were complexed with commercially available cationic lipids and then cotransfected with UV-inactivated vaccinia virus vT7-3 into CV-1 or LLMCK cells (Fuerst et al., 1986). After $40 \mathrm{~h}$, the cells were disrupted by three cycles of freezing and thawing and then injected into the chorioallantoic cavity of 10day-old embryonated chicken eggs. Subsequently, the virus was recovered, and the vaccinia virus was eliminated by a second propagation in eggs. A virus titer was determined by a hemagglutination assay using chicken red blood cells (Yonemitsu et al., 2000), and the virus was then stored at $-80^{\circ} \mathrm{C}$ until use. LM8 cells were infected with Sendai virus at an MOI $=2$ and cultured for 2-7 days until use for experiments. More than $95 \%$ cells were infected judged from $\beta$-galactosidase staining.

\section{Fluorescence microscopy}

NIH3T3 cells, Hela, and LM8 cells grown in 35-mm plates with glass coverslips were transfected using LipofectAMINE (GibcoBRL) and Fugene (Roche), respectively. Cells were fixed by incubation for $10 \mathrm{~min}$ in phosphate-buffered saline (PBS) containing 3.7\% formaldehyde and then permeabilized in PBS containing $0.1 \%$ Triton $\mathrm{X}-100$ for $5 \mathrm{~min}$ at RT. Indirect immunofluorescence analysis was carried out with the antiMyc antibody to the Myc epitope (Santa Cruz Biotechnology) and the M2 antibody to the Flag epitope (Sigma), followed by fluorescence-conjugated anti-rabbit or mouse IgG (antirabbit FITC for ZYMED, anti-mouse and rabbit Alexia for
Molecular Probes) in combination with rhodamine-conjugated phalloidin (Molecular Probes). Images were obtained by laser confocal microscopy.

\section{Immunoblot analysis}

Transient transfection, IP, and immunoblotting were performed as described previously (Sasaki et al., 2001). Interaction between Spred2 and RhoA was detected by IP and immunoblotting using anti-RhoA (Santa Cruz Biotechnology), as described (Sasaki et al., 2001; Wakioka et al., 2001). The anti-Spred 2 antibody was prepared with the KLH-conjugated peptide IKTQPPRAKSRRRKENGEC. This antibody worked well for IP but not for immunoblotting.

\section{Cell migration assays}

The modified Boyden chambers (KURABO, Japan) were used for monitoring cell migration. Essentially, overnight serumstarved cells were trypsinized and washed twice with a serumfree medium containing $0.5 \%$ BSA. Cells were plated onto the upper side of transwells (35000 cells/well) coated with fibronectin in $0.1 \mathrm{ml}$ of a serum-free medium, and $0.5 \mathrm{ml}$ of the same medium was added to the lower chamber. The cells were allowed to adhere for $1 \mathrm{~h}$. Cells were treated with the virus in a serum-free medium for $2 \mathrm{~h}$, then CCL19 or CCL2 1 was added to the lower chamber, and cells were allowed to migrate overnight at $37^{\circ} \mathrm{C}$. At the end of the experiment, the transwell inserts were washed with PBS, fixed with methanol/ acetone, and stained with hematoxylin. Cells on the upper chamber side of the membrane were removed with cotton swabs, and cells that migrated through the membrane were counted.

\section{Wound-healing assay}

To evaluate cell motility, a wound-healing assay of LM8 cell lines was performed. Cells grown to subconfluence were scraped with a sharp edge to make a cell-free area. Cells migrating into the scraped area were observed, and photographs were taken every $36 \mathrm{~h}$ after scraping in the presence of $5 \mu \mathrm{g} / \mathrm{ml}$ mitomycin $\mathrm{C}$.

\section{Nude mice xenograft experiments}

Male nude mice (6 weeks old) were purchased form Charles River Laboratories. LM8 cells were infected with the Sendai virus at an $\mathrm{MOI}=2.0$ and cultured for 2 days. Cells $\left(1 \times 10^{7}\right.$ cells) were injected subcutaneously into the flanks of nude mice. Tumor growths were monitored weekly for 3 weeks. Tumor size was determined by the product of two perpendicular diameters and the height above the skin surface.

\section{Luciferase assay}

Luciferase assays were carried out as described previously (Joo et al., 2004). Briefly, 293 cells were transfected with $0.1 \mu \mathrm{g}$ of reporter gene, $0.2 \mu \mathrm{g}$ of $\mathrm{pCH} 110$ encoding the $\beta$-galactosidase gene under the control of the SV40 promoter, and Flag- or Myc-tagged Spred expression vectors or $0.1 \mu \mathrm{g}$ V14RhoA expression vector. After $24 \mathrm{~h}$, cells were treated with or without serum $(10 \% \mathrm{FCS})$ for $6 \mathrm{~h}$ and then corrected and lysed with a PicaGene Reporter Lysis Buffer (TOYO Ink Co., Japan). The activity of luciferase and $\beta$-galactosidase was analysed by using beetle luciferin (Promega) and $o$-nitrophenyl $\beta$-galactopilanoside (Nakarai Tesq., Japan) as substrates. In all reporter assays, $2 \times 10^{5}$ of 293 cells was plated onto six-well dishes and transfected by the calcium-phosphate method. 


\section{Pull-down assay for GTP-Rho}

The 293 cells were transfected with Flag-tagged wild-type RhoA and Myc-Spred1. Pull-down assay with GST-Rhobinding domain of Rhotekin (GST-RBD) was carried out as described (Kimura et al., 2000). Briefly, after stimulation with $100 \mathrm{ng} / \mathrm{ml} \mathrm{CCL19,} 293$ cells were lysed in 1\% Nonidet P-40 in the lysis buffer. Cell lysates were clarified by centrifugation at $30000 \mathrm{~g}$ for $20 \mathrm{~min}$ and the supernatants were incubated with $30 \mu \mathrm{g}$ of GST-RBD fusion conjugated with glutathione beads at $4{ }^{\circ} \mathrm{C}$ for $2 \mathrm{~h}$. The beads were washed twice with lysis buffer and subjected to SDS-polyacrylamide gel electrophoresis on a $12 \%$ gel. Bound RhoA was detected by Western blot using a monoclonal antibody against Flag tag.

\section{Rock activity assay}

Rho-kinase activity was measured by Cyclex Rho-kinase assay kit (Cyclex Co. Ltd) according to the manufacture's instructions. Briefly, 293 cells were transfected with Myc-tagged Rock cDNA (a gift from Dr Narumiya, Kyoto University) with V14RhoA and Spred-1. After 2 days, cell extracts were immunoprecipitated with anti-Myc antibody and protein A-Sepharose. The immunoprecipitates were incubated with kinase reaction buffer containing ATP in the plates coated with a peptide of myosin-binding subunit of myosin phosphatase (MBS). After $30 \mathrm{~min}$ incubation, phosphorylation of the substrate peptide was detected with HRP-conjugated anti-phospho-MBS threonine-696-specific antibody. The reaction was quantified by spectrophotometry with absorbance at $450 \mathrm{~nm}$.

\section{Acknowledgements}

We thank Ms Kawabata-Honda and S Sata for excellent technical assistance, Dr Kaibuchi (Nagoya University) for Rho, Rac, and cdc42 plasmids, Dr Narumiya (Kyoto University) for GST-RBD and Rock plasmids, Ms Arifuku and Ms Yamaura for manuscript preparation. This work was supported by Special Grants-in-Aid from the Ministry of Education, Science, Technology, Sports, and Culture of Japan, the Japan Health Science Foundation, the Human Frontier Science Program, the Japan Research Foundation for Clinical Pharmacology, and the Uehara Memorial Foundation.

\section{References}

Amano M, Chihara K, Kimura K, Fukata Y, Nakamura N, Matsuura Y and Kaibuchi K. (1997). Science, 275, 1308-1311.

Amano M, Fukata Y and Kaibuchi K. (2000). Exp. Cell. Res., 261, 44-51.

Arsenian S, Weinhold B, Oelgeschlager M, Ruther U and Nordheim A. (1998). EMBO J., 17, 6289-6299.

Bonacchi A, Romagnani P, Romanelli RG, Efsen E, Annunziato F, Lasagni L, Francalanci M, Serio M, Laffi G, Pinzani M, Gentilini P and Marra F. (2001). J. Biol. Chem., 276, 9945-9954.

Cabrita MA and Christofori G. (2003). Thromb. Haemost., 90, 586-590.

Callebaut I, Cossart P and Dehoux P. (1998). FEBS Lett., 441, 181-185.

Carnac G, Primig M, Kitzmann M, Chafey P, Tuil D, Lamb N and Fernandez A. (1998). Mol. Cell. Biol., 9, 1891-1902.

Casci T, Vinos J and Freeman M. (1999). Cell, 96, 655-665.

De Maximy AA, Nakatake Y, Moncada S, Itoh N, Theiry JP and Bellusci S. (1999). Mech. Dev., 81, 213-216.

Fuerst TR, Niles EG, Studier FW and Moss B. (1986). Proc. Natl. Acad. Sci. USA, 83, 8122-8126.

Hacohen N, Kramer S, Sutherland D, Hiromi Y and Krasnow MA. (1998). Cell, 92, 253-263.

Hashimoto S, Nakano H, Singh G and Katyal S. (2002). Mech. Dev., 119 (Suppl 1), S303-S309.

Hill CS, Wynne J and Treisman R. (1995). Cell, 81, $1159-1170$

Impagnatiello MA, Weitzer S, Gannon G, Compagni A, Cotten M and Christofori G. (2001). J. Cell Biol., 152, 1087-1098.

Ishizaki T, Maekawa M, Fujisawa K, Okawa K, Iwamatsu A, Fujita A, Watanabe N, Saito Y, Kakizuka A, Morii N and Narumiya S. (1996). EMBO J., 15, 1885-1893.

Izawa I, Amano M, Chihara K, Yamamoto T and Kaibuchi K. (1998). Oncogene, 17, 2863-2871.

Joo A, Aburatani H, Moriii E, Iba $\mathrm{H}$ and Yoshimura A. (2004). Oncogene, 23, 726-734.

Kato A, Sakai Y, Shioda T, Kondo T, Nakanishi M and Nagai Y. (1996). Genes Cells, 1, 569-579.
Kato R, Nonami A, Taketomi T, Wakioka T, Kuroiwa A, Matsuda Y and Yoshimura A. (2003). Biochem. Biophys. Res. Commun., 302, 767-772.

Khosravi-Far R, Chrzanowska-Wodnicka M, Solski PA, Eva A, Burridge K and Der CJ. (1994). Mol. Cell. Biol., 14, 6848-6857.

Kimura K, Tsuji T, Takada Y, Miki T and Narumiya S. (2000). J. Biol. Chem., 275, 17233-17236.

Kinch MS, Clark GJ, Der CJ and Burridge K. (1995). J. Cell Biol., 130, 461-471.

Kolakofsky D, Pelet T, Garcin D, Hausmann S, Curran J and Roux L. (1998). J. Virol., 72, 891-899.

Kramer S, Okabe M, Hacohen N, Krasnow MA and Hiromi Y. (1999). Development, 126, 2515-2525.

Kranenburg O, Poland M, Gebbink M, Oomen L and Moolenaar WH. (1997). J. Cell Sci., 110, 2417-2427.

Lee SH, Schloss DJ, Jarvis L, Krasnow MA and Swain JL. (2001). J. Biol. Chem., 276, 4128-4133.

Leung T, Chen XQ, Manser E and Lim L. (1996). Mol. Cell. Biol., 16, 5313-5327.

Lim J, Wong ES, Ong SH, Yusoff P, Low BC and Guy GR. (2000). J. Biol. Chem., 275, 32837-32845.

Minowada G, Jarvis LA, Chi CL, Neubuser A, Sun X, Hacohen N, Krasnow MA and Martin GR. (1999). Development, 126, 4465-4475.

Prehoda KE, Lee DJ and Lim WA. (1999). Cell, 97, 471-480.

Reich A, Sapir A and Shilo B. (1999). Development, 126, 4139-4147.

Ridley AJ and Hall A. (1992a). Cell, 70, 389-399.

Ridley AJ, Paterson HF, Johnston CL, Diekmann D and Hall A. (1992b). Cell, 70, 401-410.

Sakai Y, Kiyotani K, Fukumura M, Asakawa M, Kato A, Shioda T, Yoshida T, Tanaka A, Hasegawa M and Nagai Y. (1999). FEBS Lett., 456, 221-226.

Sasaki A, Taketomi T, Kato R, Saeki K, Nonami A, Sasaki M, Kuriyama M, Saito N, Shibuya M and Yoshimura A. (2003). Nat. Cell Biol., 5, 427-432.

Sasaki A, Taketomi T, Wakioka T, Kato R and Yoshimura A. (2001). J. Biol. Chem., 276, 36804-36808.

Sotiropoulos A, Gineitis D, Copeland $\mathbf{J}$ and Treisman R. (1999). Cell, 98, 159-169. 
Tefft JD, Lee M, Smith S, Leinwand M, Zhao J, Bringas Jr P, Crowe DL and Warburton D. (1999). Curr. Biol., 9, 219-222.

Wakioka T, Sasaki A, Kato R, Shouda T, Matsumoto A, Miyoshi K, Tsuneoka M, Komiya S, Baron R and Yoshimura A. (2001). Nature 412. 647-651.

Wei L, Zhou W, Croissant JD, Johansen FE, Prywes R, Balasubramanyam A and Schwartz RJ. (1998). J. Biol. Chem., 273, 30287-30294.
Yigzaw Y, Cartin L, Pierre S, Scholich K and Patel TB. (2001). J. Biol. Chem., 276, 22742-22747.

Yonemitsu Y, Kitson C, Ferrari S, Farley R, Griesenbach U, Judd D, Steel R, Scheid P, Zhu J, Jeffery PK, Kato A, Hasan MK, Nagai Y, Masaki I, Fukumura M, Hasegawa M, Geddes DM and Alton EW. (2000). Nat. Biotechnol., 18, 970-973.

Zhong C, Kinch MS and Burridge K. (1997). Mol. Cell. Biol., 8, 2329-2344. 